\title{
Integral closure of ideals and annihilators of homology
}

\author{
Alberto Corso \\ Department of Mathematics, University of Kentucky, Lexington, KY 40506, USA \\ E-mail: corso@ms.uky.edu \\ Craig HuneKe \\ Department of Mathematics, University of Kansas, Lawrence, KS 66045, USA \\ E-mail: huneke@math.ukans.edu \\ DANIEL KATZ \\ Department of Mathematics, University of Kansas, Lawrence, KS 66045, USA \\ E-mail: dlk@math.ukans.edu \\ WOLMER V. VASCONCELOS \\ Department of Mathematics, Rutgers University, Piscataway, NJ 08854, USA \\ E-mail: vasconce@math.rutgers.edu
}

\section{Introduction}

Let $(R, \mathfrak{m})$ be a local Noetherian ring. Given an $R$-ideal $I$ of height $g$, a closely related object to $I$ is its integral closure $\bar{I}$. This is the set (ideal, to be precise) of all elements in $R$ that satisfy an equation of the form

$$
X^{m}+b_{1} X^{m-1}+b_{2} X^{m-2}+\cdots+b_{m-1} X+b_{m}=0,
$$

with $b_{j} \in I^{j}$ and $m$ a non-negative integer. Clearly one has that $I \subseteq \bar{I} \subseteq \sqrt{I}$, where $\sqrt{I}$ is the radical of $I$ and consists instead of the elements of $R$ that satisfy an equation of the form $X^{q}-b=0$ for some $b \in I$ and $q$ a non-negative integer. While 
[EHV] already provides direct methods for the computation of $\sqrt{I}$, the nature of $\bar{I}$ is complex. Even the issue of validating the equality $I=\bar{I}$ is quite hard and relatively few methods are known $[\mathbf{C H V}]$. In general, computing the integral closure of an ideal is a fundamental problem in commutative algebra. Although it is theoretically possible to compute integral closures, practical computations at present remain largely out-of-reach, except for some special ideals, such as monomial ideals in polynomial rings over a field. One known computational approach is through the theory of Rees algebras: It requires the computation of the integral closure of the Rees algebra $\mathcal{R}$ of $I$ in $R[t]$. However, this method is potentially wasteful since the integral closure of all the powers of $I$ are being computed at the same time. On the other hand, this method has the advantage that for the integral closure $\bar{A}$ of an affine algebra $A$ there are readily available conductors: given $A$ in terms of generators and relations (at least in characteristic zero) the Jacobian ideal Jac of $A$ has the property that Jac $\cdot \bar{A} \subseteq A$, in other words, $\bar{A} \subseteq A$ : Jac. This fact is the cornerstone of most current algorithms to build $\bar{A}[\mathbf{d e J}[\overline{\mathbf{V}}]$.

On a seemingly unrelated level, let $H_{i}=H_{i}(I)$ denote the homology modules of the Koszul complex $\mathbb{K}_{*}$ built on a minimal generating set $a_{1}, \ldots, a_{n}$ of $I$. It is well known that all the non-zero Koszul homology modules $H_{i}$ are annihilated by $I$, but in general their annihilators tend to be larger. To be precise, this article outgrew from an effort to understand our basic question:

\section{Are the annihilators of the non-zero Koszul homology modules $H_{i}$ of an unmixed ideal I contained in the integral closure $\bar{I}$ of I?}

We are particularly interested in the two most meaningful Koszul homology modules, namely $H_{1}$ and $H_{n-g}$ - the last non-vanishing Koszul homology module. Of course the case that matters most in dealing with the annihilator of the latter module is when $R$ is not Gorenstein. We also stress the necessity of the unmixedness requirement on $I$ in our question. Indeed, let $R=k[[x, y, z, w]]$ with $k$ a field characteristic zero. The ideal $I=\left(x^{2}-x y,-x y+y^{2}, z^{2}-z w,-z w+w^{2}\right)$ is an height two mixed ideal with $\operatorname{Ann}\left(H_{1}\right)=\bar{I}=(I, x z-y z-x w+y w)$ and $\operatorname{Ann}\left(H_{2}\right)=\sqrt{I}=(x-y, z-w)$. It is interesting to note that this ideal has played a significant role in [CHV], where it was shown that the integral closure of a binomial ideal is not necessarily binomial, unlike the case of its radical as shown by Eisenbud and Sturmfels [ES]. A first approach to our question would be to decide if the annihilators of the Koszul homology modules are rigid in the sense that the annihilator of $H_{i}$ is contained in the annihilator of $H_{i+1}$. Up to radical this is true by the well-known rigidity of the Koszul complex. If true, we could concentrate our attention on the last nonvanishing Koszul homology. Unfortunately, this rigidity is not true. An example was given by Aberbach: let $R=k[x, y, z] /(x, y, z)^{n+1}$ and let $E$ be the injective hull of the residue field of $R$. Then $z$ is in the annihilator of $H_{1}(x, y ; E)$, but $z^{n}$ does not annihilate $H_{2}(x, y ; E)$. It would be good to have an example where such behavior occurs for the Koszul homology of an ideal on the ring itself.

An obvious question is: What happens when $I$ is integrally closed? In Section 2 we provide some validation for our guiding question. In Corollary 2.4 we show that for any an $\mathfrak{m}$-primary ideal $I$, that is not a complete intersection, such that $c H_{1}=0$ and $c \in I: \mathfrak{m}$ for $c \in R$, then $c \in \bar{I}$. In particular, if $I$ is an integrally closed 
ideal then $\operatorname{Ann}\left(H_{1}\right)=I$. We then proceed to study ann $\left(H_{1}\right)$ for several classes of ideals with good structure: these include syzygetic ideals, height two perfect Cohen-Macaulay ideals, and height three perfect Gorenstein ideals. While in the case of height two perfect Cohen-Macaulay ideals the Koszul homology modules are faithful (see Proposition 2.10), in the case of syzygetic ideals we observe that ann $\left(H_{1}\right)$ can be interpreted as $I: I_{1}(\varphi)$, where $I_{1}(\varphi)$ is the ideal generated by the entries of any matrix $\varphi$ minimally presenting the ideal $I$ (see Proposition 2.6). In the case of height three perfect Gorenstein ideals we show the weaker statement that $\left(\operatorname{ann}\left(H_{1}\right)\right)^{2} \subset \bar{I}$ (see Theorem 2.12).

Section 3 contains variations on a result of Burch, which continue the theme of this paper in that they deal with annihilators of homology and integrally closed ideals. The result of Burch that we have in mind $[\bar{B}]$ asserts that if $\operatorname{Tor}_{t}^{R}(R / I, M)$, $M$ a finitely generated $R$-module, vanishes for two consecutive values of $t$ less than or equal to the projective dimension of $M$, then $\mathfrak{m}(I: \mathfrak{m})=\mathfrak{m} I$. This has the intriguing consequence that if $I$ is an integrally closed ideal with finite projective dimension, then $R_{\mathfrak{p}}$ is a regular local ring for all $\mathfrak{p} \in \operatorname{Ass}(R / I)$. In particular, a local ring is regular if and only if it has an m-primary integrally closed ideal of finite projective dimension. A variation of Burch's theorem is given in Theorem 3.1 We then deduce a number of corollaries. For instance, we show in Corollary 3.3 that integrally closed $\mathfrak{m}$-primary ideals $I$ can be used to test for finite projective dimension, in the sense that if $\operatorname{Tor}_{i}^{R}(M, R / I)=0$, then the projective dimension of $M$ is at most $i-1$. This improves Burch's result in that we do not need to assume that two consecutive Tors vanish. Recent work of Goto and Hayasaka ([GH1] and [GH2] ) has many more results concerning integrally closed ideals of finite projective dimension.

The annihilator of the conormal module $I / I^{2}$ is a natural source of elements in the integral closure of $I$. In Section 4 we study a class of Cohen-Macaulay ideals whose conormal module is faithful. We close with a last section giving an equivalent formulation of our main question, and also include another question which came up in the course of this study.

\section{Annihilators of Koszul homology}

We start with some easy remarks, that are definitely not sharp exactly because of their generality. It follows from localization that $\operatorname{ann}\left(H_{1}\right) \subset \sqrt{I}$. Moreover, for any $R$-ideal $I$ minimally presented by a matrix $\varphi$ we also show that $\operatorname{ann}\left(H_{1}\right) \subset I: I_{1}(\varphi)$, where $I_{1}(\varphi)$ is the ideal generated by the entries of $\varphi$. Things get sharper when one focuses on the annihilator of the first Koszul homology modules of classes of ideals with good structural properties. We conclude the section with a result of Ulrich about the annihilator of the last non-vanishing Koszul homology module.

\subsection{The first Koszul homology module}

Our first theorem is a general result about annihilators of Koszul homology. It follows from this theorem that our basic question has a positive answer for the first 
Koszul homology module in the case that $I$ is an integrally closed $\mathfrak{m}$-primary ideal.

Theorem 2.1 Let $(R, \mathfrak{m})$ be a local Noetherian ring and let I be an $\mathfrak{m}$-primary ideal. If $c \in R$ is an element such that $c H_{i}(I)=0$ then one of the following conditions hold:

(a) $I: c=\mathfrak{m} I: c$

(b) There exists $J \subseteq I$ and $x \in R$ such that $I=J+(c x), \mu(I)=\mu(J)+1$ and $c H_{i}(J)=c H_{i-1}(J)=0$.

We will need a lemma before proving Theorem 2.1

Lemma 2.2 Let $J \subseteq R$ be an ideal and $c, x \in R$. Assume that $(J, c x)$ is primary to the maximal ideal. Then $\lambda\left(H_{i}(J, c)\right)=\lambda\left(\operatorname{ann}_{c} H_{i}(J, c x)\right)$.

Proof. Induct on $i$. Suppose $i=0$. The desired equality of lengths follows immediately from the exact sequence

$$
0 \rightarrow((J, c x): c) /(J, c x) \longrightarrow R /(J, c x) \stackrel{\cdot c}{\longrightarrow} R /(J, c x) \longrightarrow R /(J, c) \rightarrow 0 .
$$

Suppose $i>0$ and the lemma holds for $i-1$. We have an exact sequence

$$
0 \rightarrow H_{i}(J, c x) / c H_{i}(J, c x) \longrightarrow H_{i}(J, c x, c) \longrightarrow \operatorname{ann}_{c}\left(H_{i-1}(J, c x) \rightarrow 0 .\right.
$$

But $H_{i}(J, c x, c)=H_{i}(J, c) \oplus H_{i-1}(J, c)$, so

$$
\lambda\left(H_{i}(J, c)\right)+\lambda\left(H_{i-1}(J, c)\right)=\lambda\left(\operatorname{ann}_{c}\left(H_{i-1}(J, c)\right)+\lambda\left(H_{i}(J, c x) / c H_{i}(J, c x)\right) .\right.
$$

Using the induction hypothesis, we obtain $\lambda\left(H_{i}(J, c)\right)=\lambda\left(H_{i}(J, c x) / c H_{i}(J, c x)\right)=$ $\lambda\left(\operatorname{ann}_{c} H_{i}(J, c x)\right)$.

Proof of Theorem 2.1 Suppose $(a)$ does not hold. Then there exists $x \in \mathfrak{m}$ such that $c x$ is a minimal generator of $I$. We can write $I=J+(c x)$, for an ideal $J \subseteq I$ satisfying $\mu(I)=\mu(J)+1$. On the one hand, from the exact sequences

$$
0 \rightarrow H_{i}(J) / c H_{i}(J) \longrightarrow H_{i}(J, c) \longrightarrow \operatorname{ann}_{c} H_{i-1}(J) \rightarrow 0
$$

and

$$
0 \rightarrow H_{i}(J) / c x H_{i}(J) \longrightarrow H_{i}(J, c x) \longrightarrow \operatorname{ann}_{c x} H_{i-1}(J) \rightarrow 0
$$

we get

$$
\lambda\left(H_{i}(J, c)\right)=\lambda\left(H_{i}(J) / c H_{i}(J)\right)+\lambda\left(\operatorname{ann}_{c} H_{i-1}(J)\right)
$$

and

$$
\lambda\left(H_{i}(J, c x)\right)=\lambda\left(H_{i}(J) / c x H_{i}(J)\right)+\lambda\left(\operatorname{ann}_{c x} H_{i-1}(J)\right) .
$$

On the other hand,

$$
\lambda\left(H_{i}(J) / c x H_{i}(J)\right) \geq \lambda\left(H_{i}(J) / c H_{i}(J)\right) \quad \text { and } \quad \lambda\left(\operatorname{ann}_{c x} H_{i-1}(J)\right) \geq \lambda\left(\operatorname{ann}_{c} H_{i}(J)\right) .
$$


Since $c H_{i}(J, c x)=0, H_{i}(J, c x)=\operatorname{ann}_{c} H_{i}(J, c x)$, so $\lambda\left(H_{i}(J, c x)\right)=\lambda\left(H_{i}(J, c)\right)$, by Lemma 2.2. It follows from this that $\lambda\left(H_{i}(J) / c H_{i}(J)\right)=\lambda\left(H_{i}(J) / c x H_{i}(J)\right)$. Thus, $c H_{i}(J)=c x H_{i}(J)$, so $c H_{i}(J)=0$, by Nakayama's lemma. Similarly, since

$$
\lambda\left(\operatorname{ann}_{c} H_{i-1}(J)\right)=\lambda\left(\operatorname{ann}_{c x} H_{i-1}(J)\right),
$$

it follows that $\lambda\left(H_{i-1}(J) / c H_{i}(J)\right)=\lambda\left(H_{i-1}(J) / c x H_{i}(J)\right)$, so $c H_{i-1}(J)=0$, as before.

Corollary 2.3 Let $(R, \mathfrak{m})$ be a local Noetherian ring and let $I$ be an $\mathfrak{m}$-primary ideal. If $c \cdot H_{1}(I)=0$, then $I: c=\mathfrak{m} I: c$.

Proof. If $I: c$ properly contains $m I: c$, then by Theorem 2.1, there exists $J \subseteq I$ and $x \in \mathfrak{m}$ such that $I=J+(c x), \mu(I)=\mu(J)+1$ and $c \cdot H_{0}(J)=0$. But then, $c \in J$, so $I=J$, a contradiction.

Corollary 2.4 Let $(R, \mathfrak{m})$ be a local Noetherian ring and let $I$ be an $\mathfrak{m}$-primary ideal. If $c \in R$ is an element such that $c \cdot H_{1}(I)=0$ and $c \in I: \mathfrak{m}$, then $c \in \bar{I}$.

Proof. Since $\mathfrak{m} \subseteq I: c$, we have $\mathfrak{m} c \subseteq \mathfrak{m} I$, by Corollary 2.3 By the determinant trick, $c \in \bar{I}$.

Corollary 2.5 Let $(R, \mathfrak{m})$ be a local Noetherian ring and let I be an integrally closed $\mathfrak{m}$-primary ideal. Then $\operatorname{Ann}\left(H_{1}\right)=I$.

Proof. Suppose ann $H_{1}(I)$ properly contains $I$. Take $c \in\left(\operatorname{ann} H_{1}(I) \backslash I\right) \cap(I: \mathfrak{m})$. By Corollary 2.4 $c \in \bar{I}=I$, a contradiction. Thus, ann $H_{1}(I)=I$.

Syzygetic ideals: It follows from the determinant trick that the annihilator of $I^{m} / I^{m+1}$ is contained in $\bar{I}$ for all $m$. Hence, another piece of evidence in support of our question is given by the close relationship between $H_{1}$ and the conormal module $I / I^{2}$. This is encoded in the exact sequence

$$
0 \rightarrow \delta(I) \longrightarrow H_{1} \longrightarrow(R / I)^{n} \longrightarrow I / I^{2} \rightarrow 0,
$$

where $\delta(I)$ denotes the kernel of the natural surjection from the second symmetric power $\operatorname{Sym}_{2}(I)$ of $I$ onto $I^{2}, \operatorname{Sym}_{2}(I) \rightarrow I^{2}$, see [SV]. We will exploit this exact sequence in at least two occasions: Proposition 2.6 and Theorem 4.1 We recall that the ideal $I$ is said to be syzygetic whenever $\delta(I)=0$.

Proposition 2.6 Let $R$ be a Noetherian ring. For any $R$-ideal I minimally presented by a matrix $\varphi, \operatorname{ann}\left(H_{1}\right) \subset I: I_{1}(\varphi)$, where $I_{1}(\varphi)$ denotes the ideal generated by the entries of $\varphi$. If, in addition, I is syzygetic then $\operatorname{ann}\left(H_{1}\right)=I: I_{1}(\varphi)$. 
Proof. Let $Z_{1}$ and $B_{1}$ denote the modules of cycles and boundaries respectively. If $x \in \operatorname{ann}\left(H_{1}\right)$ one has that for $z \in Z_{1}$ the condition $x z \in B_{1}$ means that each coordinate of $z$ is conducted into $I$ by $x$. Thus $x \in I: I_{1}(\varphi)$. The reverse containment holds if $I$ is syzygetic. In fact, in this situation one actually has that $H_{1} \hookrightarrow\left(I_{1}(\varphi) / I\right)^{n}$. Thus $I: I_{1}(\varphi) \subset \operatorname{Ann}\left(H_{1}\right)$.

Corollary 2.7 Let $R$ be a local Noetherian ring, and let I be an ideal of finite projective dimension $n$. Then $\left(\operatorname{ann}\left(H_{1}\right)\right)^{n+1} \subseteq I$.

Proof. Assume $I$ is minimally presented by a matrix $\varphi$. By the above proposition, ann $\left(H_{1}\right) \subset I: I_{1}(\varphi)$. The result then follows immediately from the following proposition of G. Levin (unpublished). The proof follows from a careful analysis of Gulliksen's Lemma, 1.3.2 in [GL].

Proposition 2.8 Let $R$ be a local Noetherian ring and let $I$ be an ideal of finite projective dimension $n$, minimally presented by a matrix $\varphi$. Then $\left(I: I_{1}(\varphi)\right)^{n+1} \subseteq I$.

Remark 2.9 In general, the ideal $I: I_{1}(\varphi)$ may be larger than the integral closure of $I$. For example the integrally closed $R$-ideal $I=(x, y)^{2}$, where $R$ is the localized polynomial ring $k[x, y]_{(x, y)}$, is such that $I: I_{1}(\varphi)=(x, y)$. However, Levin 's proposition shows that $\left(I: I_{1}(\varphi)\right)^{2} \subset I$.

Height two perfect ideals: The first case to tackle is the one of height two perfect ideals in local Cohen-Macaulay rings. However the Cohen-Macaulayness of the $H_{i}$ 's gets into the way. Indeed we have the following fact:

Proposition 2.10 Let $R$ be a local Cohen-Macaulay ring and let I be a height two perfect $R$-ideal. Then for all $i$ (with $\left.H_{i} \neq 0\right)$ one has $\operatorname{Ann}\left(H_{i}\right)=I$.

Proof. Consider the resolution of the ideal $I$

$$
0 \rightarrow R^{n-1} \longrightarrow R^{n} \longrightarrow I \rightarrow 0 \text {. }
$$

The submodule of 1 -cycles of $\mathbb{K}_{*}, Z_{1}$, is the submodule $R^{n-1}$ of this resolution. Also, for all $i$ one has $Z_{i}=\bigwedge^{i} Z_{1}$. All these facts can be traced to [AH]. This implies that for any $i \leq n-2, H_{1}^{i}=H_{i}-$ this multiplication is in $H_{*}(\mathbb{K})$. Thus the annihilator of $H_{1}$ will also annihilate, say, $H_{n-2}$. But this is the canonical module of $R / I$, and its annihilator is $I$. The conclusion now easily follows.

Gorenstein ideals: Let us consider a perfect $\mathfrak{m}$-primary Gorenstein ideal in a local Noetherian ring $R$. In this situation, if $I$ is Gorenstein but not a complete intersection then $\operatorname{Ann}\left(H_{1}\right) \neq I$. Otherwise, $R / I$ would be a submodule of $H_{1}$. By a theorem of Gulliksen [GL], if $H_{1}$ has a free summand then it must be a complete intersection. Actually, using Gulliksen's theorem one shows that if $I$ is $\mathfrak{m}$-primary, Gorenstein but not a complete intersection, then the socle annihilates $H_{1}$. Combining Proposition 2.6] and the work of $[\mathbf{C H V}]$ yields the following result: 
Proposition 2.11 Let $(R, \mathfrak{m})$ be a local Noetherian ring with embedding dimension at least 2 and let $I$ be an $\mathfrak{m}$-primary ideal contained in $\mathfrak{m}^{2}$ with $R / I$ Gorenstein. Suppose further that I is minimally presented by a matrix $\varphi$ and that $I_{1}(\varphi)=\mathfrak{m}$, where $I_{1}(\varphi)$ denotes the ideal generated by the entries of $\varphi$. Then $\operatorname{ann}\left(H_{1}\right) \subset \bar{I}$.

Proof. By Proposition 2.6 and our assumption we obtain that ann $\left(H_{1}\right) \subset I: I_{1}(\varphi)=$ $I: \mathfrak{m}$. Our assertion now follows from Lemma 3.6 in $[\mathbf{C H V}]$ since $(I: \mathfrak{m})^{2}=$ $I(I: \mathfrak{m})$.

For an height three perfect Gorenstein ideal $I$ we have some evidence that $\left(\operatorname{ann}\left(H_{1}\right)\right)^{2}=I \cdot \operatorname{ann}\left(H_{1}\right)$. If this were to hold in general, it would imply that $I \subsetneq \operatorname{ann}\left(H_{1}\right) \subset \bar{I}$. Thus far, we can prove the weaker result that the square of the annihilator of $H_{1}$ is in the integral closure of $I$.

Theorem 2.12 Let $R$ be a local Noetherian ring with $\operatorname{char}(R) \neq 2$ and let $I$ be a height three perfect Gorenstein ideal minimally generated by $n \geq 5$ elements. Then

$$
\left(\operatorname{ann}\left(H_{1}\right)\right)^{2} \subset \bar{I} .
$$

Proof. Let $a_{1}, \ldots a_{n}$ denote a set of minimal generators of $I$. Notice that $B_{1}$ and $Z_{1}$ are submodules of $R^{n}$ of rank $n-1$; in general, if $E$ is a submodule of $R^{n}$ of rank $r$, we denote by $\operatorname{det}(E)$ the ideal generated by the $r \times r$ minors of the matrix with any set of generators of $E$ (as elements of $R^{n}$ ).

Let $c \in R$ be such that $c Z_{1} \subset B_{1}$. It suffices to prove that $c^{2} \in \bar{I}$ since the square of an ideal is always integral over the ideal generated by the squares of its generators. Note that $c^{n-1} \operatorname{det}\left(Z_{1}\right) \subset \operatorname{det}\left(B_{1}\right)$. Let $V$ be a valuation overring of $R$ with valuation $v$; the ideal $I V$ is now principal and generated by one of the original generators, say $a_{1}=a$. By the structure theorem of Buchsbaum and Eisenbud [BE], we may assume that $a$ is one of the maximal Pfaffians of the matrix presenting $I$. Since $I$ is generated by $a, B_{1} V$ is generated by the Koszul syzygies $\left(a_{2},-a, 0, \ldots, 0\right),\left(a_{3}, 0,-a, \ldots, 0\right), \ldots,\left(a_{n}, 0,0, \ldots,-a\right)$. Hence $\operatorname{det}\left(B_{1} V\right)=$ $\left(a^{n-1}\right)=I^{n-1} V$. As for $Z_{1} V$, one has that $\operatorname{det}\left(Z_{1} V\right)$ includes the determinant of the minor defining $a^{2}$ ( $a$ is the Pfaffian of the submatrix). Thus $c^{n-1} I^{2} V \subset$ $I^{n-1} V$, which yields that $c^{n-1} \in I^{n-3} V$, as cancellation holds. Hence, we have that $(n-1) v(c)=v\left(c^{n-1}\right) \geq v\left(I^{n-3} V\right)=(n-3) v(I V)$. Finally, this yields

$$
v\left(c^{2}\right) \geq 2 \frac{n-3}{n-1} v(I V) \geq v(I V)
$$

and, in conclusion, $c^{2} \in \bar{I}$.

Remark 2.13 It is worth remarking that the above proof shows much more. Recall that $\overline{I^{\frac{a}{b}}}$ denotes the integral closure of the ideal generated by all $x \in R$ such that $x^{b} \in I^{a}$. From [BBE], we know that $n=2 k+1$ must be odd. Our proof shows that

$$
\left(\operatorname{ann}\left(H_{1}\right)\right) \subset \overline{I^{\frac{k-1}{k}}} .
$$

As $k$ gets large this is very close to our main objective, proving that $\left(\operatorname{ann}\left(H_{1}\right)\right) \subset \bar{I}$. 


\subsection{Last non-vanishing Koszul homology module}

Let us turn our attention towards the tail of the Koszul complex.

Proposition 2.14 Let $R$ be a one-dimensional domain with finite integral closure. Then any integrally closed ideal is reflexive. In particular, for any ideal I its bidual $\left(I^{-1}\right)^{-1}$ is contained in its integral closure $\bar{I}$.

Proof. We may assume that $R$ is a local ring, of integral closure $B$. An ideal $L$ is integrally closed if $L=R \cap L B$. Since $B$ is a principal ideals domain, $L B=x B$ for some $x$. We claim that $x B$ is reflexive. Let $C=B^{-1}=\operatorname{Hom}_{R}(B, R)$ be the conductor of $B / R$. $C$ is also an ideal of $B, C=y B$, and therefore $C^{-1}=y^{-1} B^{-1}=y^{-1} C$, which shows that $C^{-1}=B$. This shows that $\left(L^{-1}\right)^{-1} \subset\left(R^{-1}\right)^{-1} \cap\left((x B)^{-1}\right)^{-1}=L$. The last assertion follows immediately by setting $I \subset L=\bar{I}$.

We can interpret the above result as an annihilation of Koszul cohomology. Let $I=\left(a_{1}, \ldots, a_{m}\right)$ and let $\mathbb{K}^{*}$ denote the Koszul complex

$$
0 \rightarrow R \longrightarrow R^{m} \longrightarrow \bigwedge^{2} R^{m} \longrightarrow \cdots \longrightarrow \bigwedge^{m} R^{m} \rightarrow 0
$$

with differential $\partial(w)=z \wedge w$, where $z=a_{1} e_{1}+\cdots+a_{n} e_{m}$. One sees that $Z^{1}=I^{-1} z$, and $B^{1}=R z$. Thus $\left(I^{-1}\right)^{-1}$ is the annihilator of $H^{1}$. On the other hand $H^{1} \cong$ $H_{m-1} \cong \operatorname{Ext}_{R}^{1}(R / I, R)$. Let us raise a related issue: $\left(I^{-1}\right)^{-1}$ is just the annihilator of $\operatorname{Ext}_{R}^{1}(R / I, R)$, so one might want to consider the following question which is obviously relevant only if the ring $R$ is not Gorenstein. Let $R$ be a Cohen-Macaulay geometric integral domain and let $I$ be a height unmixed ideal of codimension $g$. Is $\operatorname{ann}\left(\operatorname{Ext}_{R}^{g}(R / I, R)\right)=\operatorname{ann}\left(H_{n-g}\right)$ always contained in $\bar{I}$ ? Notice that the annihilator of the last non-vanishing Koszul homology can be identified with $J:(J: I)$ for $J$ an ideal generated by a maximal regular sequence inside $I$. This follows since the last non-vanishing Koszul homology is isomorphic to $(J: I) / J$.

We thank Bernd Ulrich for allowing us to reproduce the following result [U], which grew out of conversations at MSRI (Berkeley):

Theorem 2.15 (Ulrich) Let $(R, \mathfrak{m})$ be a Cohen-Macaulay local ring, let I be an $\mathfrak{m}$-primary ideal and let $J \subset I$ be a complete intersection. Then $J:(J: I) \subset \bar{I}$. In particular the annihilator of the last non-vanishing Koszul homology of $I$ is contained in the integral closure of I.

Proof. We may assume that ht $J=$ ht $I$. We may also assume that $R$ has a canonical module $\omega$. We first prove:

Lemma 2.16 Let $A$ be an Artinian local ring with canonical module $\omega$ and let $I \subset A$ be an ideal. Then $0:_{\omega}\left(0:_{A} I\right)=I \omega$. 
Proof. Note that $0:_{\omega}\left(0:_{A} I\right)=\omega_{R / 0: I}$. To show $I \omega=\omega_{R / 0: I}$ note that the socle of $I \omega$ is 1 -dimensional as it is contained in the socle of $\omega$. Hence we only need to show that $I \omega$ is faithful over $R / 0: I$. Let $x \in \operatorname{ann}_{R} I \omega$, then $x I \omega=0$, hence $x I=0$, hence $x \in 0: I$.

Returning to the proof of Theorem 2.15 it suffices to show that $(J:(J: I)) \omega \subset I \omega$. But $(J:(J: I)) \omega \subset J \omega:_{\omega}\left(J:_{R} I\right)$. So it suffices to show $J \omega:_{\omega}\left(J:_{R} I\right) \subset I \omega$. Replacing $R, \omega$ by $R / J, \omega_{R / J}=\omega / J \omega$ we have to show $0:_{\omega}\left(0:_{R} I\right) \subset I \omega$, which holds by Lemma 2.16

\section{Variations on a theorem of Burch}

Theorem 3.1 below is a variation of Burch's theorem mentioned in the introduction, and strengthens it in the case $I$ is integrally closed. We then deduce a number of corollaries.

Theorem 3.1 Let $(R, \mathfrak{m})$ be a local ring, I an integrally closed $R$-ideal having height greater than zero and $M$ a finitely generated $R$-module. For $t \geq 1$, set $J_{t}:=$ $\operatorname{ann}\left(\operatorname{Tor}_{t}(R / I, M)\right)$. Let $\left(\mathbb{F}_{*}, \varphi_{i}\right)$ be a minimal free resolution of $M$. If image $\left(\varphi_{t}\right)$ is contained in $\varnothing \mathfrak{m} J_{t} F_{t-1}$, then

$$
\operatorname{image}\left(\varphi_{t} \otimes_{R} 1_{R / I}\right) \cap \operatorname{socle}\left(F_{t-1} / I F_{t-1}\right)=0 .
$$

Proof. Take $u \in F_{t-1}$ such that its residue class modulo $I$ belongs to

$$
\operatorname{image}\left(\varphi_{t} \otimes_{R} 1_{R / I}\right) \cap \operatorname{socle}\left(F_{t-1} / I F_{t-1}\right) \text {. }
$$

Then $u=\varphi_{t}(v)+w$, for $v \in F_{t}$ and $w \in I F_{t-1}$. For all $x \in \mathfrak{m}, \varphi_{t}(x v) \equiv 0$ modulo $I F_{t-1}$. Thus for all $j \in J_{t}$, there exists $z \in F_{t+1}$ such that $\varphi_{t+1}(z) \equiv j x v$ modulo $I F_{t}$. It follows that we can write $j x v=\varphi_{t+1}(z)+w_{0}$, for $w_{0} \in I F_{t}$. Therefore, $j x u=\varphi_{t}(j x v)+j x w=\varphi_{t}\left(w_{0}\right)+j x w$. By hypothesis, we get $j x u \in \emptyset m J_{t} I F_{t}$, for all $j \in J_{t}$ and all $x \in \mathfrak{m}$. Therefore, by cancellation, $u \in I_{a} F_{t-1}$. But since $I$ is integrally closed, $u \in I F_{t-1}$, which gives what we want.

In the following corollaries, we retain the notation from Theorem 3.1

Corollary 3.2 Suppose I is integrally closed and $\mathfrak{m}$-primary. If image $\left(\varphi_{t}\right)$ is contained in $\emptyset \mathfrak{m} J_{t} F_{t-1}$, then:

(a) $\operatorname{image}\left(\varphi_{t}\right) \subseteq I F_{t-1}$.

(b) $J_{t} F_{t} \subseteq$ image $\left(\varphi_{t+1}\right)$.

Proof. For $(a)$, if image $\left(\varphi_{t} \otimes 1_{R / I}\right)$ were not zero, then it would contain a non-zero socle element, since $I$ is $\mathfrak{m}$-primary. This contradicts Theorem 3.1 so $(a)$ holds. 
For $(b)$, it follows from $(a)$ that $\operatorname{Tor}_{t}(R / I, M)=F_{t} /\left(\operatorname{image}\left(\varphi_{t+1}\right)+I F_{t}\right)$, so $J_{t} F_{t}$ is contained in image $\left(\varphi_{t+1}\right)+I F_{t}$, and $(b)$ follows via Nakayama's Lemma.

The next corollary shows that integrally closed $\mathfrak{m}$-primary ideals can be used to test for finite projective dimension.

Corollary 3.3 Suppose that I is integrally closed and $\mathfrak{m}$-primary. Then $M$ has projective dimension less than $t$ if and only if $\operatorname{Tor}_{t}(R / I, M)=0$.

Proof. The hypothesis implies that $J_{t}=R$. Therefore, image $\left(\varphi_{t}\right)$ is automatically contained in $\emptyset \mathfrak{m} J_{t} F_{t-1}$. By part $(b)$ of Corollary 3.2 $F_{t} \subseteq \operatorname{image}\left(\varphi_{t+1}\right)$, so $F_{t}=0$, by Nakayama's Lemma.

Corollary 3.4 Let $J \subseteq R$ be an ideal and I an integrally closed $\mathfrak{m}$-primary ideal. If $J \subseteq \emptyset \mathfrak{m}(I J: I \cap J)$, then $J \subseteq I$.

Proof. Apply Corollary 3.2 with $M=R / J$ and $t=1$.

Corollary 3.5 Suppose that $R$ is reduced and $M$ has infinite projective dimension over $R$. Then for all $t \geq 1$, the entries of $\varphi_{t}$ do not belong to $\emptyset \mathfrak{m} \cdot \operatorname{ann}(M)$. In particular, each map in the minimal resolution of $k$ has an entry not belonging to $\varnothing \mathfrak{m}^{2}$.

Proof. Let $I$ be any $\mathfrak{m}$-primary integrally closed ideal. If the entries of $\varphi_{t}$ belong to $\emptyset \mathfrak{m} \cdot \operatorname{ann}(M)$, then image $\left(\varphi_{t}\right)$ is contained in $\varnothing \mathfrak{m} J_{t} F_{t-1}$. By Corollary 3.2 image $\left(\varphi_{t}\right)$ is contained in $I F_{t-1}$. But the intersection of the integrally closed $\mathfrak{m}$-primary ideals is zero, therefore, image $\left(\varphi_{t}\right)=0$, contrary to the hypothesis on $M$. Thus, the conclusion of the corollary holds.

The last statement follows in the case $R$ is regular from the fact that the Koszul complex on a minimal set of generators of the maximal ideal gives a resolution of $k$. If $R$ is not regular, then $k$ has infinite projective dimension, and the result follows at once from the first statement.

In regard to the above corollary, it is well-known that the Koszul complex of a minimal set of generators of the maximal ideal is part of a minimal resolution of $k$ in all cases, so for the maps occuring in the minimal resolution up to the dimension of the ring, the last statement is clear. The new content of the last statement is for the maps past the dimension of the ring.

Corollary 3.6 Suppose I is integrally closed and $\mathfrak{m} \in \operatorname{Ass}(R / I)$. If image $\left(\varphi_{t}\right)$ is contained in $\emptyset \mathfrak{m} J_{t} F_{t-1}$, e.g., $\operatorname{Tor}_{t}(R / I, M)=0$, then either $M$ has projective dimension less than $t-1$ or $\mathfrak{m} \in \operatorname{Ass}\left(\operatorname{Tor}_{t-1}(R / I, M)\right)$.

Proof. Suppose $M$ has projective dimension greater than or equal to $t-1$. Then $F_{t-1} \neq 0$. By hypothesis, the socle of $F_{t-1} / I F_{t-1}$ is non-zero, so a non-zero element $u$ in this socle goes to zero under $\varphi_{t-1} \otimes 1_{R / I}$. But the theorem implies that the image of $u$ in $\operatorname{Tor}_{t-1}(R / I, M)$ remains non-zero, so the result holds. 


\section{The conormal module}

We end the article with a result in the spirit of our investigation. More precisely we show that the conormal module $I / I^{2}$ is faithful for a special class of CohenMacaulay ideals.

Theorem 4.1 Let $(R, \mathfrak{m})$ be a Gorenstein local ring and I a Cohen-Macaulay almost complete intersection. Let $\varphi$ be a matrix minimally presenting I. If $I_{1}(\varphi)$ is a complete intersection, then $I / I^{2}$ is a faithful $R / I$-module.

Proof. Let $g$ denote the height of $I$, write $n=g+1$ for the minimal number of generators of $I=\left(a_{1}, \ldots, a_{n}\right)$. We may assume that the ideals generated by any $g$ of the $a_{i}$ 's are complete intersection ideals. Let $e_{i}$, with $1 \leq i \leq n$, denote the $n$-tuple $(0, \ldots, 0,1,0, \ldots, 0)$ where 1 is in the $i$-th position. Finally, note that $H_{1}$ is the canonical module of $R / I$.

Let us consider the exact sequence

$$
0 \rightarrow \delta(I) \longrightarrow H_{1} \stackrel{\theta}{\longrightarrow}(R / I)^{n} \stackrel{\pi}{\longrightarrow} I / I^{2} \rightarrow 0,
$$

where $\delta(I)$ is the kernel of the natural surjection $\operatorname{Sym}_{2}(I) \rightarrow I^{2}$, see $[\mathbf{S V}]$. Notice that for any $\varepsilon^{\prime}=\sum r_{j}^{\prime} e_{j}+B_{1} \in H_{1}$, where $\sum r_{j}^{\prime} a_{j}=0$, one has $\theta\left(\varepsilon^{\prime}\right)=\left(r_{1}^{\prime}+I\right) e_{1}+$ $\ldots+\left(r_{n}^{\prime}+I\right) e_{n}$ while for any element in $(R / I)^{n}$ one has $\pi\left(\left(r_{1}+I\right) e_{1}+\ldots+\left(r_{n}+\right.\right.$ $\left.I) e_{n}\right)=r_{1} a_{1}+\ldots+r_{n} a_{n}+I^{2}$. Apply $(-)^{\vee}=\operatorname{Hom}_{R / I}\left(-, H_{1}\right)$ to the above exact sequence. We obtain

$$
0 \rightarrow\left(I / I^{2}\right)^{\vee} \stackrel{\pi^{\vee}}{\longrightarrow} \operatorname{Hom}\left((R / I)^{n}, H_{1}\right) \stackrel{\theta^{\vee}}{\longrightarrow} \operatorname{Hom}\left(H_{1}, H_{1}\right)=R / I \longrightarrow \delta(I)^{\vee} \rightarrow 0 .
$$

To conclude it will be enough to show that $\left(I / I^{2}\right)^{\vee}$ is faithful.

First, we claim that the image of $\theta^{\vee}$ belongs to $I_{1}(\varphi) / I$. In fact, any element of $\operatorname{Hom}\left((R / I)^{n}, H_{1}\right)$ can be written as a combination of elementary homomorphism of the form

$$
\xi_{i}\left((1+I) e_{i}\right)=\varepsilon \quad \xi_{i}\left((1+I) e_{j}\right)=0, \quad \text { if } i \neq j,
$$

with $\varepsilon=\sum r_{j} e_{j}+B_{1} \in H_{1}$, where $\sum r_{j} a_{j}=0$. Thus, for any $\varepsilon^{\prime} \in H_{1}$ we have

$$
\left(\theta^{\vee}\left(\xi_{i}\right)\right)\left(\varepsilon^{\prime}\right)=\xi_{i}\left(\theta\left(\varepsilon^{\prime}\right)\right)=\xi_{i}\left(\sum\left(r_{j}^{\prime}+I\right) e_{j}\right)=\left(r_{i}^{\prime}+I\right) \varepsilon .
$$

Observe that $\left(r_{i}^{\prime}+I\right) \varepsilon=\left(r_{i}+I\right) \varepsilon^{\prime}$ in $H_{1}$. Indeed, $r_{i}^{\prime} \varepsilon-r_{i} \varepsilon^{\prime}=\sum\left(r_{i}^{\prime} r_{j}-r_{i} r_{j}^{\prime}\right) e_{j}+B_{1}$. But $\sum\left(r_{i}^{\prime} r_{j}-r_{i} r_{j}^{\prime}\right) e_{j}$ is a syzygy of the complete intersection $\left(a_{1}, \ldots, \widehat{a}_{i}, \ldots, a_{n}\right)$ and thus it is a Koszul syzygy of the smaller ideal: hence it is in $B_{1}$. In conclusion, $\theta^{\vee}\left(\xi_{i}\right)$ is nothing but multiplication by $r_{i}+I \in I_{1}(\varphi) / I$. Given that $\varepsilon$ and $i$ were chosen arbitrarily one has that the image of $\theta^{\vee}$ is $I_{1}(\varphi) / I$.

Notice that the number of generators of $I_{1}(\varphi)$ is strictly smaller than $n$. So we can say that the image of $\theta^{\vee}$ is given say by $\left(\theta^{\vee}\left(\xi_{2}\right), \ldots, \theta^{\vee}\left(\xi_{n}\right)\right)$. Write, for some $c_{i} \in R / I$,

$$
\theta^{\vee}\left(\xi_{1}\right)=\sum_{i \geq 2} c_{i} \theta^{\vee}\left(\xi_{i}\right)
$$


Hence $\xi_{1}-\sum_{i \geq 2} c_{i} \xi_{i} \in \operatorname{Ker}\left(\theta^{\vee}\right)=\operatorname{Im}\left(\pi^{\vee}\right)$ so that we can find $\gamma \in\left(I / I^{2}\right)^{\vee}$ such that

$$
\xi_{1}-\sum_{i \geq 2} c_{i} \xi_{i}=\pi^{\vee}(\gamma)=\gamma \circ \pi
$$

The restriction of these homomorphisms to the first component of $\operatorname{Hom}\left((R / I)^{n}, H_{1}\right)$ gives an homomorphism from $R / I$ to $H_{1}$. Now, something that annihilates $\gamma$ would also annihilate the restriction, but that restriction is faithful.

Remark 4.2 From the proof of Theorem 4.1 we also obtain that $\operatorname{Hom}\left(\delta(I), H_{1}\right)=$ $R / I_{1}(\varphi)$. In addition, if $I_{1}(\varphi)$ is Cohen-Macaulay of codimension $g$ then by the theorem of Hartshorne-Ogus we have that $\delta(I)$ (which is $S_{2}$ ) is Cohen-Macaulay and therefore depth $I / I^{2} \geq d-g-2$.

Unfortunately, there is not much hope to stretch the proof of Theorem 4.1 as the following example shows.

Example 4.3 Let $R$ be the localized polynomial ring $k[x, y]_{(x, y)}$. The ideal $I=$ $\left(x^{5}-y^{5}, x^{4} y, x y^{4}\right)$ is such that $I^{2}: I=\left(I, x^{3} y^{3}\right)$. In this case $I_{1}(\varphi)=(x, y)^{2}$ so that $\mu(I)=\mu\left(I_{1}(\varphi)\right)=3$.

\section{More Questions}

We end by considering some other closely related questions which came up during the course of this investigation. We let $I$ be an $\mathfrak{m}$-primary ideal of the local ring $R$ minimally generated by $n$ elements, and let $J_{i}$ be the annihilator of the $i$ th Koszul cohomology of $I$ with respect to a minimal generating set of $I$.

$$
\text { Set } d \text { equal to the dimension of } R \text {. Is } J_{1} \cdot J_{2} \cdots J_{n-d} \text { contained in } \overline{I^{n-d}} \text { ? }
$$

Notice that the Koszul homology of $I$ vanishes for values larger than $n-d$, so that the product above represents all the interesting annihilators of the Koszul homology of $I$. Furthermore, a postive answer to this question gives a positive answer to our main question. This follows since each $J_{i}$ contains $I$. Along any discrete valuation $v$, this means that $v(I) \geq v\left(J_{i}\right)$ for all $i$. A positive answer to the question above implies that

$$
\sum_{i=1}^{n-d} v\left(J_{i}\right) \geq(n-d) v(I) \geq \sum_{i=1}^{n-d} v\left(J_{i}\right)
$$

It would follow that $v\left(J_{i}\right)=v(I)$ for all $i$, implying that $J_{i} \subseteq \bar{I}$ for all $1 \leq i \leq n-d$. Conversely, if $J_{i} \subseteq \bar{I}$ for all $1 \leq i \leq n-d$, then clearly $J_{1} \cdot J_{2} \cdots J_{n-d}$ is contained 
in $\overline{I^{n-d}}$, so the above question is equivalent to saying that $J_{i} \subseteq \bar{I}$ for all $1 \leq i \leq$ $n-d$. This form of the question suggests using homotopies to compare the Koszul complex of a set of generators of $I$ with the free resolution of $I$. However, we have not been able to use this idea to settle the question.

Another question which arose during our work is the following:

Let $n$ be the number of minimal generators of an $\mathfrak{m}$-primary ideal I in a Cohen-Macaulay local ring $R$ with infinite residue field, and let $d$ be the dimension of the ring. For every $j, d \leq j \leq n-1$, choose $j$ general minimal generators of $I$, and let $J_{j}$ be the ideal they generate. Let $H_{n-j}$ denote the $(n-j)$ th Koszul homology of a minimal set of generators of I. Is

$$
\operatorname{Ann}\left(H_{n-j}\right) \subseteq J_{j}:\left(J_{j}: I\right) \subseteq \bar{I} ?
$$

We have positive answers to this question for the two extremes: $j=d$ and $j=n-1$, in the latter case assuming $I$ is integrally closed.

\section{Acknowledgments}

C. Huneke and W.V. Vasconcelos gratefully acknowledge partial support from the National Science Foundation.

\section{References}

[AH] L. Avramov and J. Herzog, The Koszul algebra of a codimension 2 embedding, Math. Z. 175 (1980), 249-260.

[BE] D. Buchsbaum and D. Eisenbud, Algebra structures for finite free resolutions, and some structure theorems for ideals of codimension 3, Amer. J. Math. 99 (1977), 447-485.

[B] L. Burch, On ideals of finite homological dimension in local rings, Math. Proc. Camb. Phil. Soc. 74 (1968), 941-948.

[CHV] A. Corso, C. Huneke and W.V. Vasconcelos, On the integral closure of ideals, Manuscripta Math. 95 (1998), 331-347.

[deJ] T. de Jong, An algorithm for computing the integral closure, J. Symbolic Comput. 26 (1998), 273-277.

[EHV] D. Eisenbud, C. Huneke and W.V. Vasconcelos, Direct methods for primary decomposition, Invent. Math 110 (1992), 207-235.

[ES] D. Eisenbud and B. Sturmfels, Binomial ideals, Duke Math. J. 84 (1996), $1-45$.

[GH1] S. Goto and F. Hayasaka, Finite homological dimension and primes associated to integrally closed ideals, Proc. Amer. Math. Soc. 130 (2002), $3159-3164$. 
[GH2] S. Goto and F. Hayasaka,Finite homological dimension and primes associated to integrally closed ideals II, J. Math. Kyoto Univ. 42 (2002), 631-639.

[GL] T.H. Gulliksen and G. Levin, Homology of Local Rings, Queen's Papers in Pure and Applied Math. 200, Queen's University, Kingston, 1967.

[SV] A. Simis and W.V. Vasconcelos, The syzygies of the conormal module, American J. Math. 103 (1981), 203-224.

[U] B. Ulrich, Personal communication.

[V] W.V. Vasconcelos, Computing the integral closure of an affine domain, Proc. Amer. Math. Soc. 113 (1991), 633-638. 